\title{
Soroprevalência para hepatite A e hepatite B em quatro centros no Brasil
}

\author{
Hepatitis A and hepatitis B seroprevalence in \\ four centers in Brazil
}

\begin{abstract}
Sue Ann Costa Clemens ${ }^{1,2}$, José Carlos Da Fonseca ${ }^{3}$, Tânia Azevedo ${ }^{4}$, Anamaria Cavalcanti ${ }^{5}$, Thêmis R. Silveira ${ }^{6}$, Marcia C. Castilho ${ }^{3}$ e Ralf Clemens ${ }^{2}$
\end{abstract}

Resumo Avaliou-se a prevalência de anticorpos para as hepatites $A$ e $B$ em 3.653 indivíduos, em quatro regiões brasileiras. As prevalências de anti-VHA e de anti-HBc foram $64,7 \%$ e 7,9\%, respectivamente. Prevalências mais elevadas de anti-VHA $(92,8 \%)$ e de anti-HBc $(21,4 \%)$ foram observadas na região Norte. Em outras regiões, prevalências de anti-VHA acima de $90 \%$ foram alcançadas apenas em idades mais avançadas, indicando uma endemicidade intermediária e prevalência significativamente mais elevada foi observada no grupo de baixo nível sócioeconômico, entre 1 e 30 anos. Para o anti-HBc observou-se um aumento na prevalência entre adolescentes e uma prevalência significativamente mais elevada no grupo de baixo nível sócioeconômico, entre 1 e 20 anos. Prevalência de 3,1\% foi encontrada em crianças de 1 ano, sugerindo a transmissão vertical. Os principais resultados deste estudo, indicam que pré-adolescente/ adolescentes de algumas cidades brasileiras estão em risco para as hepatites $A$ e $B$, mas por diferentes motivos.

Palavras-chaves: Hepatite A. Hepatite B. Epidemiologia. Soroprevalência.

Abstract The prevalence of antibodies to hepatitis $A$ and $B$ virus was assessed in 3,653 subjects across four regions of Brazil. The anti-HAV and anti-HBc seroprevalence were $64.7 \%$ and $7.9 \%$, respectively. The highest anti-HAV (92.8\%) and anti-HBc $(21.4 \%)$ rates were seen in the Northern region. In other regions, anti-HAV seroprevalence over $90 \%$ was only reached in the more elderly, indicating an intermediate endemicity and a significantly higher anti-HAV prevalence was seen in the low socioeconomic group between 1-30 years. With respect to anti-HBc seroprevalence an increase was seen in adolescents and there was a significantly higher anti-HBc prevalence in the lower socioeconomic group between 1-20 years. A 3.1\% anti-HBC prevalence was seen in oneyear-old infants, suggesting a vertical transmission. The major findings of this study indicate that the pre-adolescent and adolescent population in some Brazilian cities are at greatest risk from both hepatitis $A$ and $B$ infection, but for different reasons.

Key-words: Hepatitis A. Hepatitis B. Epidemiology. Seroprevalence.

Em todo mundo, as hepatites A e B continuam a ser um grande problema de saúde para as sociedades. O Brasil tem diversas características de países em rápido desenvolvimento, como a migração de comunidades rurais para as cidades, o que tem levado a formação de áreas urbanas de baixa renda e socialmente carentes. Contudo, essas cidades em expansão mantêm

\footnotetext{
1. Instituto de Pós-graduação Médica Carlos Chagas; 2. SmithKline Beecham Biologicals, Brasil; 3. Instituto de Medicina Tropical do Amazonas/Gerência de Virologia; 4. Universidade Federal Fluminense; 5. Hospital Infantil Albert Sabin; 6. Hospital de Clínicas de Porto Alegre.

Suporte financeiro: SmithKline Beecham Biologicals

Endereço para correspondência:Dra Sue Ann Costa Clemens. SmithKline Beecham, Brasil. Av. Comandante Guaranys 447; Jacarepaguá, 227756-610 Rio de Janeiro, RJ, Brasil

Fax: 0 xx $21445-5656$

Recebido para publicação em 19/10/98.
} 
comunidades ricas que se sobrepõem à áreas de baixa renda. Sob tais condições, várias doenças, em particular as doenças transmissíveis, podem impor um grande dispêndio econômico. Embora as vias de transmissão do vírus da hepatite $\mathrm{A}$ (VHA) e do vírus da hepatite $B$ (VHB) sejam diferentes, a disseminação de qualquer doença dentro da população é influenciada pelas condições ambientais prevalentes.

Informações atualizadas sobre a prevalência de anticorpos contra doenças imunopreveníveis são essenciais para o desenvolvimento de estratégias de saúde pública, como campanhas de vacinação. Assim, este estudo teve por objetivo avaliar a soroepidemiologia das hepatites A e B em 4 regiões brasileiras distintas: Norte, Nordeste, Sudeste e Sul. A prevalência de anticorpos para o VHA e o VHB foi determinada para toda a população estudada, e também estratificada por sexo, idade e classe sócioecônomica.

\section{MATERIAL E MÉTODOS}

Entre abril de 1996 e maio de 1997 foi realizado um estudo soroepidemiológico, transversal, multicêntrico e baseado na população em 6 países latino-americanos: Brasil, Argentina, Chile, República Dominicana, México e Venezuela. No Brasil, os locais do estudo situavam-se na região Norte (Manaus, $\mathrm{AM}$ ), na região Nordeste (Fortaleza, CE), na região Sudeste (Rio de Janeiro e Nova Friburgo, RJ) e na região Sul (Porto Alegre, $\mathrm{RS})$. Foram incluídos no estudo voluntários de ambos os sexos, entre 1 e 40 anos de idade. No Amazonas, os indivíduos foram recrutados em vilas não atingidas pela vacinação contra hepatite B. Voluntários da comunidade, de Universidades e de escolas foram recrutados em Fortaleza. No Estado do Rio de Janeiro, foram incluídos no estudo voluntários recrutados na comunidade, em Universidades, escolas, ambulatórios, consultórios e entre doadores de sangue. Voluntários da comunidade e de escolas participaram do estudo em Porto Alegre. Indivíduos com renda familiar mensal inferior a 3 salários mínimos foram classificados como pertencentes a classe sócio-econômica baixa e aqueles com renda igual ou superior a 3 salários mínimos foram incluídos na classe sócioeconômica média/alta.

O cálculo do tamanho amostral baseou-se nas recomendações da Organização Mundial de Saúde para inquéritos epidemiológicos ${ }^{27}$. Um maior número de indivíduos foi recrutado no Rio de Janeiro a fim de permitir a determinação de diferenças sócio-econômicas e, na faixa etária de 30-40 anos, recrutou-se aproximadamente metade do número de indivíduos incluídos em outras faixas etárias. A coleta de dados, utilizando-se questionários estruturados, foi realizada pelos investigadores e pessoal médico auxiliar.
O objetivo primário do estudo foi avaliar a soroprevalência de anticorpos anti-VHA, como marcador da infecção pelo VHA, e anticorpos anti-HBc, como marcador da infecção pelo VHB. Os objetivos secundários foram avaliar a soroprevalência de anticorpos anti-VHA e anti$\mathrm{HBc}$ de acordo com sexo, região e nível sócioeconômico.

O protocolo da pesquisa foi aprovado, em cada centro de pesquisa, pelas respectivas Comissões de Ética. O consentimento escrito e informado, antes do ingresso no estudo e de qualquer procedimento, foi obtido de todos os participantes (ou de seus pais/responsáveis). $\mathrm{O}$ estudo foi conduzido de acordo com as normas atuais de Boas Práticas Clínicas.

Após o consentimento informado e por escrito, obtiveram-se amostras de soro que foram armazenadas a $-20^{\circ} \mathrm{C}$ até a análise em laboratório qualificado e certificado. Anticorpos do tipo IgG contra o VHA foram detectados usando-se um kit de imunoensaio enzimático comercialmente disponível (HAVAB EIA, Abbott Laboratories, Chicago, IL, USA). Anticorpos anti-HBc, do tipo IgG, foram determinados por ELISA (Corzyme, Laboratórios Abbott). Para o VHA e VHB valores acima de $33,0 \mathrm{mlU} / \mathrm{ml}$ e $1,0 \mathrm{mIU} / \mathrm{ml}$ foram considerados positivos, respectivamente. Os dados foram colocados no Dbase IV e a análise estatística descritiva foi realizada usando-se os programas SPSS e Epilnfo 6.04. A análise estatística comparativa foi realizada usando-se o teste exato de Fisher bicaudal, com nível a de 0,05 . O intervalo de confiança de $95 \%$ (IC de 95\%) para proporções foram computados usando-se uma distribuição assintótica.

\section{RESULTADOS}

Demografia. Um total de 8.866 indivíduos foram recrutados nos seis países latino- americanos participantes do estudo. Desses, 3.879 foram recrutados no Brasil, sendo que 226 
foram excluídos por amostras de sangue perdidas/não analisáveis $(n=49)$, formulários de relato de casos com informações incompletas ou não disponíveis e violação do protocolo $(n=177)$. Os dados demográficos da população estudada, estratificados por sexo, nível sócio-econômico e idade são mostrados nas Tabelas 1 e 2. Em 3 dos 4 centros, a estratificação por idade estabelecida no delineamento do estudo foi seguida adequadamente. Também, a distribuição por sexo foi igualmente equilibrada em todos os grupos etários, exceto no grupo de 16 a 20 anos, onde cerca de 2/3 eram mulheres e 1/3 eram homens (dados não publicados).

Hepatite A. A soroprevalência geral para o VHA no Brasil foi de 64,7\% (IC de 95\% = 63,2-

Tabela 1 - Dados demográficos - distribuição por sexo e classe sócio-econômica.

\begin{tabular}{|c|c|c|c|c|c|c|c|c|c|}
\hline \multirow{3}{*}{ Região } & \multirow{3}{*}{ Noo } & \multicolumn{4}{|c|}{ Sexo } & \multicolumn{4}{|c|}{ Classe sócio-econômica } \\
\hline & & \multicolumn{2}{|c|}{ masculino } & \multicolumn{2}{|c|}{ feminino } & \multicolumn{2}{|c|}{ alta/média } & \multicolumn{2}{|c|}{ baixa } \\
\hline & & $\mathrm{n}^{\circ}$ & $\%$ & $\mathrm{n}^{\circ}$ & $\%$ & $\mathrm{n}^{\circ}$ & $\%$ & $\mathrm{n}^{\circ}$ & $\%$ \\
\hline Sudeste & 2090 & 1000 & 47,8 & 1090 & 52,2 & 1229 & 58,8 & 861 & 41,2 \\
\hline Norte & 613 & 338 & 55,1 & 275 & 44,9 & 264 & 43,1 & 349 & 56,9 \\
\hline Nordeste & 489 & 226 & 46,2 & 263 & 53,8 & 216 & 44,2 & 273 & 55,8 \\
\hline Sul & 461 & 209 & 45,3 & 252 & 54,7 & 246 & 53,4 & 215 & 46,6 \\
\hline Brasil (total) & 3653 & 1773 & 48,5 & 1880 & 51,5 & 1955 & 53,5 & 1698 & 46,5 \\
\hline
\end{tabular}

Tabela 2 - Dados demográficos - distribuição por faixa etária.

\begin{tabular}{lcccccccc}
\hline Região & $\begin{array}{c}\text { Total } \\
n^{\circ}\end{array}$ & $\begin{array}{c}1-5 \text { anos } \\
n^{\circ}\end{array}$ & $\begin{array}{c}6-10 \text { anos } \\
n^{\circ}\end{array}$ & $\begin{array}{c}11-15 \text { anos } \\
n^{\circ}\end{array}$ & $\begin{array}{c}16-20 \text { anos } \\
n^{\circ}\end{array}$ & $\begin{array}{c}21-30 \text { anos } \\
n^{\circ}\end{array}$ & $\begin{array}{c}31-40 \text { anos } \\
n^{\circ}\end{array}$ & $\begin{array}{c}\text { Média de idade } \\
\text { (anos } \pm \text { DP) }\end{array}$ \\
\hline Sudeste & 2090 & 394 & 383 & 402 & 416 & 308 & 187 & $14,9(9,8)$ \\
Norte & 613 & 72 & 161 & 38 & 51 & 202 & 89 & $18,1(10,6)$ \\
Nordeste & 489 & 99 & 99 & 95 & 93 & 51 & 52 & $14,7(9,8)$ \\
Sul & 461 & 90 & 83 & 86 & 105 & 64 & 33 & $14,6(9,4)$ \\
\hline Brasil (total) & 3653 & 655 & 726 & 621 & 665 & 625 & 361 & $15,4(10,0)$ \\
\hline
\end{tabular}

$\mathrm{DP}=$ desvio padrão

66,3). O padrão de soroprevalência foi, contudo, muito heterogêneo. Soroprevalência alta foi encontrada na região Norte (Manaus: $92,8 \%$, IC de $95 \%=90,8-94,9)$ e na região Nordeste (Fortaleza: $76,5 \%$, IC de $95 \%=72,7-80,2$ ), enquanto endemicidades menores (55,7\%) foram encontradas nas regiões Sul (Porto Alegre: IC de $95 \%=51,2-60,3$ ) e Sudeste (Rio de Janeiro: IC de $95 \%=53,6-57,8$ ) (Tabela 3).

Não houve diferença na prevalência de anticorpos anti-VHA entre os sexos masculino $(64 \%)$ e feminino $(65,9 \%)$ na população estudada $(p=0.391)$ (dados não publicados).

A Tabela 4 mostra a soroprevalência, por grupo etário. Uma baixa soroprevalência de anticorpos anti-VHA foi encontrada em crianças. A soroprevalência de anticorpos anti-VHA atingiu níveis superiores a $90 \%$ somente em coortes mais velhas, indicando um desvio para um padrão de endemicidade intermediária. Estratificação adicional dos dados por idade e por grupo sócio-econômico, mostrou uma soroprevalência significativamente maior para o anti-VHA no grupo sócio-econômico mais baixo em todos os grupos etários, exceto entre os indivíduos de idade mais avançada (Tabela 5). Esta diferença foi vista em todas as regiões, com exceção da região Norte, onde já se observou alta soroprevalência de anticorpos anti-VHA na infância em ambas as classes sócio-econômicas (Figuras 1a-1d).

Hepatite B. A soroprevalência geral de anticorpos anti-HBc no Brasil foi de 7,9\% (IC de $95 \%=7,0-8,7)$. Não observou-se diferença significativa na prevalência de anticorpos antiHBc entre homens (8,9\%; IC de 95\% = 7,6-10,2) e mulheres $(6,9 \%$; IC de $95 \%=5,8-8,0)$.

A soroprevalência de anticorpos anti-HBc em cada uma das 4 regiões estudadas é mostrada na Tabela 6 . A maior taxa foi observada na região Norte $(21,4 \%$; IC de $95 \%=18,1-24,6)$, e a menor na região Nordeste $(1,2 \%$; IC de $95 \%=0,3-2,2)$. 
Tabela 3 - Prevalência de anticorpos anti-VHA nos diferentes centros do estudo.

\begin{tabular}{lrrrc}
\hline & & \multicolumn{3}{c}{ Anti-VHA positivo } \\
\cline { 3 - 5 } Região & № & $n^{\circ}$ & $\%$ & IC de 95\% \\
\hline Sudeste & 2090 & 1164 & 55,7 & $53,6-57,8$ \\
Norte & 613 & 569 & 92,8 & $90,8-94,9$ \\
Nordeste & 489 & 374 & 76,5 & $72,7-80,2$ \\
Sul & 461 & 257 & 55,7 & $51,2-60,3$ \\
\hline Brasil (Total) & 3653 & 2364 & 64,7 & $63,2-66,3$ \\
\hline
\end{tabular}

№ = número total em cada centro; ํㅡ = número total de positivos; IC de 95\% = intervalo de confiança de 95\%; VHA = vírus da hepatite A.

Tabela 4 - Prevalência de anticorpos anti-VHA por idade.

\begin{tabular}{lcccc}
\hline $\begin{array}{l}\text { Idade } \\
\text { (anos) }\end{array}$ & Total & \multicolumn{3}{c}{ anti-VHA positivo } \\
\cline { 3 - 5 } $1-5$ & & $\mathrm{n}^{\mathbf{0}}$ & $\%$ & IC de $95 \%$ \\
$6-10$ & 655 & 230 & 35,1 & $31,5-38,8$ \\
$11-15$ & 726 & 391 & 53,9 & $50,2-57,5$ \\
$16-20$ & 621 & 377 & 60,7 & $56,9-64,5$ \\
$21-30$ & 665 & 485 & 72,9 & $69,6-76,3$ \\
$31-40$ & 625 & 537 & 85,9 & $83,2-88,6$ \\
\hline Total & 361 & 344 & 95,3 & $93,1-97,5$ \\
\hline
\end{tabular}

nº = numero em cada faixa etária; IC de 95\% = intervalo de confiança de 95\%

Tabela 5 - Prevalência de anticorpos anti-VHA por idade e classe sócio-econômica.

\begin{tabular}{|c|c|c|c|c|c|c|c|c|c|}
\hline \multirow{4}{*}{$\begin{array}{l}\text { Idade } \\
\text { (anos) }\end{array}$} & \multirow{4}{*}{ Total } & \multicolumn{7}{|c|}{ Classe sócio-econômica } & \\
\hline & & \multicolumn{4}{|c|}{ alta/média } & \multicolumn{4}{|c|}{ baixa } \\
\hline & & \multicolumn{4}{|c|}{ Anti-HAV positivo } & \multicolumn{4}{|c|}{ Anti-HAV positivo } \\
\hline & & № & $\mathrm{n}^{\circ}$ & $\%$ & IC de $95 \%$ & № & $\mathrm{n}^{\circ}$ & $\%$ & IC de $95 \%$ \\
\hline $1-5$ & 655 & 280 & 48 & 17,1 & $12,7-21,6$ & 375 & 182 & 48,5 & $43,5-53,6$ \\
\hline $6-10$ & 726 & 311 & 93 & 29,9 & $24,8-35,0$ & 415 & 298 & 71,8 & $67,5-76,1$ \\
\hline $11-15$ & 621 & 327 & 168 & 51,4 & $46,0-56,8$ & 294 & 209 & 71,1 & $65,9-76,3$ \\
\hline $16-20$ & 665 & 428 & 278 & 65,0 & $60,4-69,5$ & 237 & 207 & 87,3 & $83,1-91,6$ \\
\hline $21-30$ & 625 & 401 & 324 & 80,8 & $76,9-84,7$ & 224 & 213 & 95,1 & $92,3-97,9$ \\
\hline $31-40$ & 361 & 208 & 197 & 94,7 & $91,7-97,8$ & 153 & 147 & 96,1 & $93,0-99,2$ \\
\hline Total & 3653 & 1955 & 1108 & 56,7 & $54,5-58,9$ & 1698 & 1256 & 74,0 & $71,9-76,1$ \\
\hline
\end{tabular}

№ = número total em uma classe sócio-econômica específica; ํㅡ= número de soropositivos; IC de 95\% = intervalo de confiança de 95\%

Tabela 6 - Prevalência de anticorpos anti-HBc por centro.

\begin{tabular}{|c|c|c|c|c|}
\hline \multirow[b]{2}{*}{ Região } & \multirow[b]{2}{*}{ № } & \multicolumn{3}{|c|}{ anti-Bc positivo } \\
\hline & & $\mathrm{n}^{0}$ & $\%$ & IC de $95 \%$ \\
\hline Sudeste & 2090 & 115 & 5,5 & $4,5-6,5$ \\
\hline Norte & 613 & 131 & 21,4 & $18,1-24,6$ \\
\hline Nordeste & 489 & 6 & 1,2 & $0,3-2,2$ \\
\hline Sul & 461 & 35 & 7,6 & $5,2-10,0$ \\
\hline Brasil (Total) & 3653 & 287 & 7,9 & $7,0-8,7$ \\
\hline
\end{tabular}

№ = número total em cada centro; ํㅡ= número total de soropositivos; IC de $95 \%$ = intervalo de confiança de $95 \%$ 

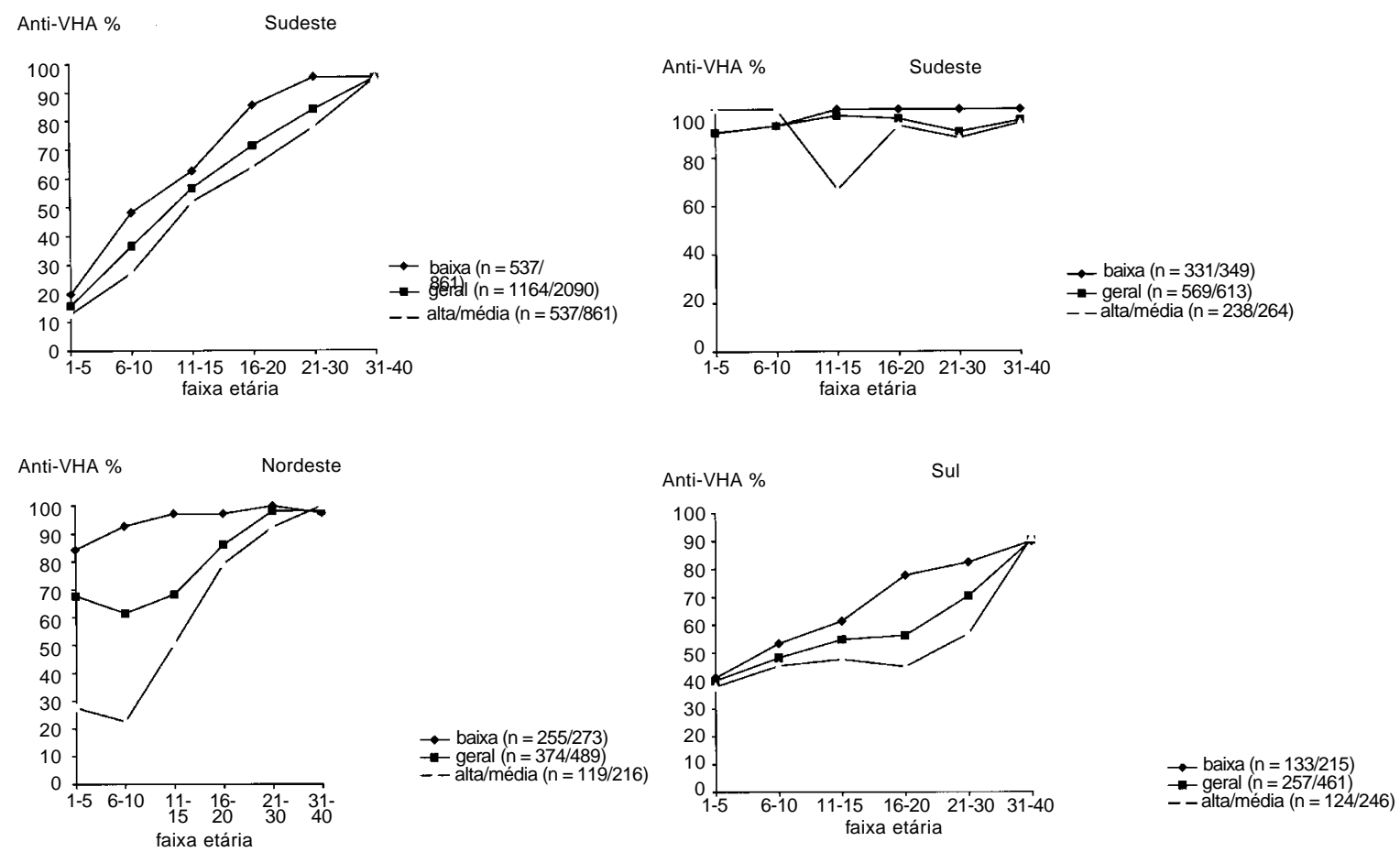

Figura 1 - Prevalência de anticorpos anti-VHA por idade, classe sócio-econômica e centro.

As regiões Sudeste e Sul mostraram taxas intermediárias de soroprevalência $(5,5 \%$; IC de $95 \%=4,5-6,5$ e $7,6 \%$; IC de $95 \%=5,2-10,0$, respectivamente).

A Tabela 7 mostra a soroprevalência de anticorpos anti-HBc estratificada por faixa etária. A infecção pelo VHB já é observada em idades precoces, com uma soroprevalência de anti-HBc de 3,1\% em crianças de um ano de idade. Além disso, há um aumento significativo na taxa de infecção de adolescentes para adultos jovens, com a prevalência de anticorpos anti-HBc quase triplicando. Também, estratificando por situação sócio-econômica observou-se uma diferença estatisticamente significante nas taxas de soroprevalência de anti-HBc entre as classes

Tabela 7 - Prevalência de anticorpos anti-HBc por idade.

\begin{tabular}{lcccc}
\hline & & \multicolumn{3}{c}{ Anti-HBc positivo } \\
\cline { 3 - 5 } idade (anos) & Total & no & \% & IC de 95\% \\
\hline 1 & 129 & 4 & 3,1 & $0,1-6,1$ \\
$2-10$ & 1252 & 79 & 6,3 & $5,0-7,7$ \\
$11-15$ & 621 & 30 & 4,8 & $3,1-6,5$ \\
$16-20$ & 665 & 37 & 5,6 & $3,8-7,3$ \\
$21-30$ & 625 & 77 & 12,3 & $9,7-14,8$ \\
$31-40$ & 361 & 60 & 16,6 & $12,7-20,4$ \\
\hline Total & 3653 & 287 & 7,9 & $6,9-8,7$ \\
\hline
\end{tabular}

$\mathrm{n}^{\circ}=$ número em cada faixa etária; IC de $95 \%$ = intervalo de confiança de $95 \%$ 
sócio-econômicas alta/média e baixa até a idade de 20 anos (Tabela 8).

Os resultados de soroprevalência de anticorpos anti-HBc estratificados por grupos etários e sócio-econômicos, em cada uma das quatro regiões estudadas, são mostrados na Figura 2. Aumento significativo na soroprevalência de anticorpos anti-HBc, entre adolescentes e adultos jovens, foi observado no Rio de Janeiro, Porto Alegre e Fortaleza. Esta tendência observou-se nos grupos sócio-econômicos alto/ médio e baixo. Em quase todas as faixas etárias, uma soroprevalência mais elevada foi observada na classe sócio-econômica baixa. Em Manaus, uma alta soroprevalência de anticorpos anti-HBc já foi encontrada na infância, no grupo sócioeconômico mais baixo. Na classe sócioeconômica média/alta observou-se baixa soroprevalência na infância que aumentou consideravelmente na adolescência.

Tabela 8 - Prevalência de anticorpos anti-HBc por idade e classe sócio-econômica.

\begin{tabular}{|c|c|c|c|c|c|c|c|c|c|c|}
\hline \multirow{4}{*}{$\begin{array}{l}\text { Idade } \\
\text { (anos) }\end{array}$} & \multirow[b]{4}{*}{ Total } & \multicolumn{8}{|c|}{ Classe socio-econômica } & \multirow[b]{4}{*}{$\mathrm{p}$ (valor) } \\
\hline & & \multicolumn{4}{|c|}{ alta/média } & \multicolumn{4}{|c|}{ baixa } & \\
\hline & & \multicolumn{4}{|c|}{ Anti-HBc positivo } & \multicolumn{4}{|c|}{ Anti-HBc positivo } & \\
\hline & & № & $\mathrm{n}^{0}$ & $\%$ & IC de $95 \%$ & № & $\mathrm{n}^{\mathrm{o}}$ & $\%$ & IC de $95 \%$ & \\
\hline $1-10$ & 1381 & 591 & 9 & 1,5 & $0,5-2,5$ & 790 & 74 & 9,4 & $7,3-11,4$ & $<0,001$ \\
\hline $11-15$ & 621 & 327 & 4 & 1,2 & $0,3-2,4$ & 294 & 26 & 8,8 & $5,6-12,1$ & $<0,001$ \\
\hline $16-20$ & 665 & 428 & 15 & 3,5 & $1,8-5,2$ & 237 & 22 & 9,3 & $5,6-13,0$ & 0,002 \\
\hline $21-30$ & 625 & 401 & 44 & 11,0 & $7,9-14,0$ & 224 & 33 & 14,7 & $10,1-19,4$ & 0,204 \\
\hline $31-40$ & 361 & 208 & 34 & 16,3 & $11,3-21,4$ & 153 & 26 & 17,0 & $11,0-22,9$ & 0,887 \\
\hline Total & 3653 & 1955 & 106 & 5,4 & $4,4-6,4$ & 1698 & 181 & 10,7 & $9,2-12,1$ & $<0,001$ \\
\hline
\end{tabular}

№ = número total dentro de uma classe sócio-econômica específica; $\mathrm{n}^{\circ}=$ número de soropositivos; IC de 95\% = intervalo de confiança de $95 \%$

Anti-HBC \% Sudeste

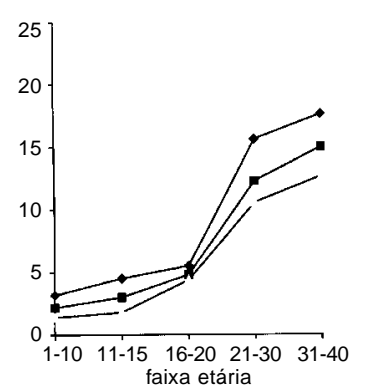

Anti-HBC \% Nordeste

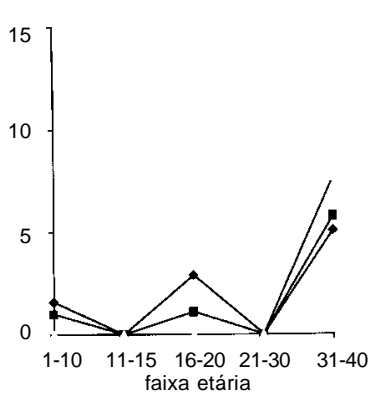

Anti-HBC \% Norte

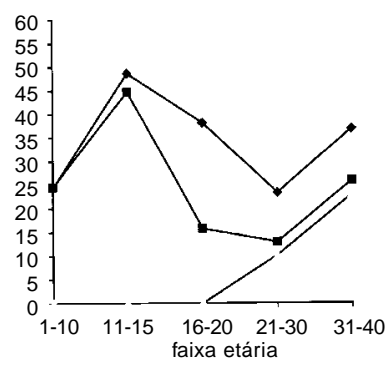

$\rightarrow$ baixa $(n=98 / 349)$ - geral $(n=131 / 613)$ baixa $(n=59 / 861)$

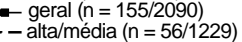

- baixa $(n=5 / 273)$ - alta/média $(n=1 / 216)$

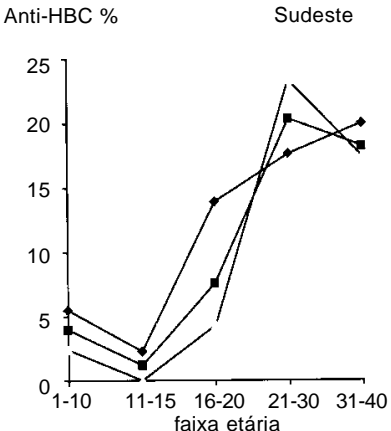

$\rightarrow$ baixa $(n=19 / 215)$

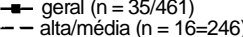

Figura 2 - Prevalência de anticorpos anti-HBc por idade, classe sócio-econômica e centro. 


\section{DISCUSSÃO}

Existem diferenças muito evidentes no Brasil em termos geográficos, climáticos, econômicos e na origem étnica da população e todas essas diferenças interferem na epidemiologia das doenças. Neste estudo, mais de 3.600 pessoas entre 1 e 40 anos de idade foram recrutadas em 4 diferentes regiões do país: região Norte (Manaus, $\mathrm{AM}$ ); região Nordeste (Fortaleza, $\mathrm{CE}$ ); região Sudeste (Rio de Janeiro e Nova Friburgo, RJ) e região Sul (Porto Alegre, RS). Esses quatro centros foram escolhidos para abranger a heterogenicidade observada na população brasileira.

Na América Latina, estudos epidemiológicos recentes sobre a hepatite $A$ têm mostrado, em diversos países, uma mudança de endemicidade alta para intermediária ${ }^{10}$. Embora a população estudada seja heterogênea, observou-se uma mudança na soroprevalência do HAV para endemicidade intermediária nas regiões Nordeste, Sul e Sudeste. Isto pode estar relacionado ao desenvolvimento econômico ocorrido nos últimos 20 anos. Houve também uma diferença evidente na soroprevalência de anticorpos anti-VHA de acordo com os níveis sócio-econômicos, com taxas significativamente menores nas classes sociais alta/média, refletindo provavelmente melhores condições de higiene. Em contraste, a população da região Norte exibiu, típicamente, um padrão de alta endemicidade.

A diversidade de soroprevalência de anticorpos anti-VHA na população representa um problema de saúde pública. As cidades da América Latina são, típicamente, compostas de populações com níveis sócios-econômicos alto e baixo, residindo em áreas muito próximas umas das outras. Igualmente, áreas rurais circundando as cidades são relativamente pobres em comparação com as populações urbanas próximas. Assim, crianças, adolescentes e adultos jovens soronegativos têm um risco similar ao de viajantes para regiões de alta endemicidade, considerando que eles não estão protegidos, porém estão sob risco contínuo de exposição. Sob tais condições ocorrem surtos epidêmicos com conseqüências potencialmente graves para indivíduos de maior idade e que não estão protegidos pela imunidade natural. $\mathrm{Na}$ Argentina, estudo recente mostrou que $64 \%$ dos casos de insuficiência hepática fulminante em crianças, requerendo transplante hepático, estavam ligados a infecção pelo $\mathrm{VHA}^{6}$. Além disso, a infecção em crianças é agora considerada mais grave do que previamente se pensava, devido especialmente à probabilidade e conseqüências da insuficiência hepática fulminante ${ }^{15}$.

Sem dúvida, melhorias adicionais na higiene e, em particular, no suprimento de uma água segura seriam a longo prazo os meios de reduzir a soroprevalência de anticorpos anti-VHA no Brasil. Contudo, a curto prazo, as diferentes taxas de desenvolvimento sócio-econômico criam uma situação paradoxal, na qual algumas populações tem alto risco da infecção e doença clínica. Experiências prévias tem demonstrado que a vacinação, é capaz de evitar a disseminação da doença durante surtos ${ }^{1417}$ e também proteger contactantes domiciliares ${ }^{19}$. Por esta razão a vacinação contra o VHA é o meio mais efetivo de controle da doença sendo, conseqüentemente, o recurso de saúde pública de escolha. Além disso, o custo-benefício da vacinação contra o VHA está claramente ilustrado pelo despêndio econômico que foi imposto, recentemente, à comunidade de Puglia na Itália ${ }^{13}$, onde um surto de VHA envolvendo 5.889 pessoas custou 37.406 milhões de dólares (662 dólares por paciente).

Um estudo recente no Brasil mostrou que, em populações com endemicidade intermediária para o VHA, a média de idade em que ocorre a infecção é de 6,7 anos ${ }^{28}$. Portanto, a vacinação das crianças, antes do seu ingresso na escola, poderia conferir proteção antes da sua exposição ao VHA, sendo que esta estratégia de vacinação deveria ser preferida. Além disso, as crianças são a principal fonte de infecção para seus pais e responsáveis, nos quais a evolução clínica da doença é mais grave. Em populações onde a endemicidade está modificando-se, a idade dos indivíduos susceptíveis continuará a aumentar e conseqüentemente a população alvo para vacinação. Em termos de proteção para a população de maior idade, pode-se considerar que a soroprevalência é suficientemente baixa em adultos jovens e que o pré-teste não seria custo-efetivo ${ }^{10}$.

Neste estudo, foi observado um padrão intermediário de endemicidade para a hepatite $B$, no Brasil. Embora as taxas de soroprevalência de anticorpos anti-HBc tenham sido menores do que as encontradas em trabalhos anteriores, onde somente populações adultas foram 
consideradas, elas são comparáveis quando apenas a população adulta é analisada ${ }^{724}$. Além disso, o fato do recrutamento neste estudo ter sido voluntário pode significar que uma população de menor risco para a hepatite $B$ foi recrutada. Entretanto, observou-se nas quatro regiões brasileiras um amplo espectro de taxas de soroprevalência de anticorpos anti-HBc. A Bacia Amazônica foi descrita previamente como sendo uma região de alta endemicidade ${ }^{24}$ e compatível com este dado, encontrou-se uma soroprevalência de $21,4 \%$ na região Norte. Igualmente, em concordância com relatos anteriores, foram observadas menores taxas de soroprevalência no sul do país $(5,5 \%$ e $7,6 \%$ no Rio de Janeiro e Porto Alegre, respectivamente $)^{28}$.

A estratificação da população estudada, por faixa etária, mostrou que $3,1 \%$ das crianças eram soropositivas para anticorpos anti-HBc com um ano de idade, sugerindo a transmissão vertical. A esse respeito, vale ressaltar que aproximadamente um-quarto (24,5\%) das crianças entre 1 e 10 anos eram soropositivas para anticorpos anti-HBc em Manaus, o que é típico de uma região de alta endemicidade onde a transmissão vertical e a horizontal precoce são importantes vias de infecção. Nas quatro regiões, foi observado um aumento significativo na soroprevalência para o VHB na adolescência, indicando que a atividade sexual é uma significativa via de transmissão deste agente, nesta população ${ }^{21}$. Na América Latina, como em outras regiões do mundo, existe freqüentemente uma falta de informação social e cultural sobre a hepatite $B$, exceto entre grupos de alto risco, como usuários de drogas endovenosas.

No Brasil, áreas de alta endemicidade, como a Bacia Amazônica Ocidental, tem sido alvo de programas de vacinação para lactentes ${ }^{9}$. Desde então, dois-terços de todas as crianças desta região completaram um esquema de três doses de vacinação contra o VHB ${ }^{24}$. A Bacia Amazônica Ocidental tem uma grande população de indígenas. Esta população isolada também tem sido associada com uma alta soroprevalência para o vírus de hepatite D (VHD). O VHD é dependente do VHB para sua replicação ${ }^{18}$. Como resultado, a soroprevalência de anticorpos anti-VHD tende a se correlacionar com a do VHB. Embora a infecção pelo VHB seja predominantemente perinatal, o VHD é transmitido por via sexual, mais tarde durante a vida 35121625 .
A superinfecção pelo VHD tem sido implicada como causa de diversos surtos de hepatite grave na Bacia Amazônica brasileira. A gravidade da hepatite resultante da infeção pelo VHD foi uma razão adicional para se priorizar os programas de vacinação neonatal na Amazônia. Pensava-se que a infecção pelo VHD fosse restrita a população ameríndia, contudo, recentemente ela parece estar aumentando em outras cidades da América do Sul24, possivelmente devido a migração de áreas rurais. Portanto, a vacinação de adolescentes pode tornar-se cada vez mais importante para prevenir a transmissão do VHD na população.

Dada a importância da transmissão vertical e o impacto da transmissão sexual nas taxas de soroprevalência de anticorpos anti-VHB observadas neste estudo, sugere-se que lactentes, adolescentes e adultos jovens são os segmentos da população de maior risco, especialmente, em áreas urbanas. Estudos na Itália demonstraram o benefício da vacinação de neonatos e adolescentes de 12 anos de idade ${ }^{4}$. Esta dupla estratégia de vacinação levará a uma cobertura vacinal da população entre as idades de 0 a 24 anos dentro de 12 anos e após este período somente neonatos precisarão ser vacinados.

Com o objetivo de facilitar a implementação de programas de vacinação em massa contra o VHB, a Organização Mundial de Saúde tem recomendado combinar vacinas contra o VHB com outras vacinas obrigatórias na infância como, por exemplo, a vacina contra difteria, tétano e pertussis (DTPw) ${ }^{20}$. As vacinas combinadas DTPw-hepatite B e DTPw-hepatite B-Hib estão agora disponíveis e, não só facilitarão a implementação de programas de vacinação contra o VHB, mas também, poderão reduzir significativamente os custos com 0 armazenamento e transporte associados com os programas de vacinação na infância ${ }^{11}$. Recentemente, foi licenciada no Brasil uma vacina combinada contra as hepatites $A$ e $B^{22} 23$ para uso em crianças e adultos. Assim, agora existe a possibilidade de com uma única vacina, proteger-se contra ambas as doenças. Esta vacina combinada poderia ser utilizada em crianças e adolescentes, especialmente em grupos de nível sócio-econômico médio e alto e poderia oferecer a solução ideal para garantir a proteção antes do período de maior risco.

\section{AGRADECIMENTOS}

Os autores agradecem a Marc Fourneau, Gabriela Fernández, Luis Gonzalez-Urban e Magda Luna assim como também
Miranda Crichton e Norbert De Clercq pelas análises estatísticas e auxílio na preparação deste artigo. 


\section{REFERÊNCIAS BIBLIOGRÁFICAS}

1. Azevedo T, Costa S, Silveira T. Prevalence of hepatitis A antibodies in Rio de Janeiro - Brazil. In: Abstract of $1^{\text {st }}$ World Congress of Pediatric Infectious Diseases, $15^{\text {th }}$ International Congress of Pediatric Infectious Diseases, Acapulco, Gro. Mexico 4-7 December, E203. 1996.

2. Bensabath BS, Boshell J. Presença do antígeno Austrália em populações do interior do Estado do Amazonas, Brazil. Revista do Instituto de Medicina Tropical de São Paulo 15:284-288, 1973.

3. Bensabath G, Hadler SC, Soares MCP, Fields H, Dias LB, Popper H, Maynard JE. Hepatitis delta virus infection and Lebrea hepatitis. Prevalence and role in fulminant hepatitis in the Amazon Basin. Journal American Medical Association 258:479-483, 1987.

4. Bonanni P. Implementation in Italy of a universal vaccination programm against hepatitis $B$. Vaccine 13:S68-71, 1995.

5. Centers for Disease Control. Enterically transmitted nonA, non-B hepatitis Mexico Morbidity and Mortality Weekly Report 36:241-243, 1987.

6. Ciocca M, Ramonet M, Cuarterolo M, Sashon J, Garcia de Davilla MT, Alvarez E, Albano L, Speranza AM, Morise S, Gomez S, Debbag R, Imventarza O, Garrahan JP. Fulminant hepatic failure for viral hepatitis in children. In: Abstract of Proceedings $9^{\text {th }}$ Triennial International Symposium on Viral Hepatitis and Liver Disease, Rome, vol 125. A310. 1996.

7. Fay $\mathrm{OH}$. Hepatitis B in Latin America: Epidemiological patterns and eradication strategy. Vaccine 8: S100-S106, 1990

8. Ferreira CRB, Yoshida CFT, Mercadante LAC, Gomes DF, Oliveria JM, Franca MS, Sidoni M, Ennes IC, Baptista ML, Schatzmair HG, Gaspar AMC. Immunization against hepatitis $B$ in children from endemic zone: evaluation of the antibody response against DNA recombinant vaccine (Engerix B-20mcg). Revista do Instituto de Medicina Tropical de São Paulo 35:89-92, 1993.

9. Fonseca JC, Brasil LM, Braga WSM. In: Abstract of Informe actual sobre el programma de vacunaión contra el VHB en al estado de Amazonas, Brazil. GEN 46 242, 1992.

10. Gaspar AMC, Vitral CL, Lemos ERS, Santos DCM, Trinta KS, Sidoni M, Oliveira JM, Yoshida CFT. Epidemiology of Hepatitis A in Rio de Janeiro: A changing pattern is emerging. In: Abstract of $9^{\text {th }}$ Triennal International Symposium on Viral Hepatitis and Liver Disaese, Rome Italy 21-25 april, A53. 1996.

11. Hadler SC. Cost benefit of combining antigens. Biologicals 22:415-418, 1994.

12. Hadler SC, Monzon M, Bensabath G, Martinez Duran M, Schatz G, Fields HA Epidemiology of hepatitis delta virus infection in less developed countries. Progress in Clinical and Biological Research 364:21-31, 1991.
13. Lucioni C, Cipriani V, Mazzi S, Panunzio M. Cost of an outbreak of hepatitis A in Publia, Italy. Pharmaeconomics 13:257-266, 1998

14. McMahon BJ, Beller M, Williams J, Schloss M, Tanttila $H$ and Bulkow LA. Program to control an outbreak of Hepatitis A in Alaska by using an inactivated hepatitis A vaccine. Archives of Pediatrics \& Adolescent Medicine 150:733-739, 1996.

15. Moreira-Silva SF, Frauches DO, Pereira FE. Hepatite fulminante em crianças em Vitória, ES: observação em Hospital pediátrico no período 1992-97. Revista da Sociedade Brasileira de Medicina Tropical 31 (supl 1):87, 1998.

16. Popper H, Thung SN, Gerber MA, Hadler SC, de Monzon M, Ponzetto A, Anzola E, Rivera D, Mondolfi A, Bracho A. Histological studies of severe delta agent infection in Venezuelan Indians. Hepatology 3:906-912, 1983.

17. Prikazsky V, Olear V, Cernoch A, Safary A and André FE. Interruption of an outbreak of hepatitis $A$ in two villages by vaccination. Journal of Medical Virology 44:457-459, 1994.

18. Robinson WS. Hepatitis $B$ virus and hepatitis $D$ virus. In: Mandell GD, Benett JE, Dolin R (ed) Principles and practice of infectious diseases. Churchill Livingston, New York. p 1406-1439, 1995.

19. Sagliocca L, Amoroso P, Stroffolini T, Adamo B, Tosti ME, Lettieri G, Esposito C, Buonocore S, Pierri P, Mele A. Efficacy of hepatitis $A$ vaccine in prevention of secondary hepatitis A infection: a randomised trial. Lancet 353:113639, 1999.

20. Special Advisory Group of Experts (SAGE) report . Weekly Epidemiological Record 71: 263-264, 1996.

21. Szmuness W, Much WM, Prince AM. On the role of sexual behavior in the spread of the hepatitis B infection. In: Annals of Internal Medicine 83:489, 1975.

22. Tholen S, Van Damme P, Leentvaar-Kuypers A, LerouxRoels G, Bruguera M, Frei PC, Bakasenas B, Safary A. The first combined vaccine against hepatitis $A$ and $B$ : an overview. Vaccine 17:1657-62, 1999.

23. Thompson SC, Norris M. Immunogenicity and reactogenicity of a combined hepatitis A-Hepatitis B vaccine in adolescents. International Journal of Infectious Diseases 2:193-196, 1998.

24. Torres JR. Hepatitis $B$ and hepatitis delta virus infection in South America. Gut 38:S48-S55, 1996.

25. Torres JR and Mondolfi A. Protracted outbreak of severe delta hepatitis: experience in an isolated Amerindian population in the Upper Orinoco Basin. Reviews of Infectious Diseases 13:52-55, 1991.

26. World Health Organization. Informal discussion on quadrivalent diphtheria-tetanus-pertussis-hepatitis B vaccine. Final report. Geneva, May, 1992. 
27. World Scientific Group. Multipurpose serological surveys and World Health Organization serum reference banks. Geneva: WHO Technical Report Serie 454, 1970.
28. Zanetta DMT, Azevedo-Neto RS, Silveria ASB, Nokes DJ. Seroprevalence of hepatitis $A$ in a community of Sao Paulo, Brazil. In: Abstract of $7^{\text {th }}$ International Congress for Infectious Disease Hong Kong 10-13 June, 1996. 